\section{El tabaquismo en la mujer augura aumento de enfermedad coronaria}

La Organización Mundial de la Salud ha expresado el temor de que el aumento del hábito de fumar en mujeres de 35 a 64 años de edad en muchos países a la larga se acompañe de una mayor incidencia de infarto del miocardio en la mujer. Esta inquietud obedece a un análisis preliminar de los resultados obtenidos en los últimos 10 años del proyecto MONICA (del inglés MONItoring CArdiovascular disease), que es el ensayo multicéntrico más grande que jamás se haya llevado a cabo sobre la enfermedad coronaria. La conclusión se basa en el estudio de 150000 casos de infarto del miocardio y de 180000 fichas de individuos con factores de riesgo.

Según los resultados, las tasas de enfermedad coronaria muestran una tendencia descendente en general, quizá menos marcada en algunos países de Europa Oriental y en la China. Simultáneamente, las probabilidades de sobrevivir después de un infarto del miocardio son mucho mayores que antes gracias a los nuevos tratamientos. No obstante, los beneficios son más marcados en los hombres que en las mujeres, cuyas tasas de enfermedad coronaria están bajando con más lentitud. Estas tendencias podrían guardar una relación con el aumento progresivo del número de mujeres que fuman.

Cuando se examinaron otros factores de riesgo de enfermedad coronaria, se detectó una tendencia descendente de la tensión arterial en casi todas las poblaciones. En cambio, no hay tendencias uniformes en la reducción del hábito de fumar. Las diferencias en muchos países dependen de la edad y el sexo. En casi todos los países, las concentraciones séricas de colesterol siguen siendo demasiado altas. Se llegó a la conclusión, sobre la base de los datos obtenidos en la última década, que hoy en día las concentraciones de colesterol son similares a las de hace 10 años en todas partes con la única excepción de la China, donde se observan concentraciones bajas o en los límites de la normalidad. El estudio confirmó que la obesidad es un factor de riesgo importante. En casi todas las poblaciones se encontró un aumento importante del número de hombres y mujeres con obesidad.

El proyecto MONICA de la OMS ha estado a la vanguardia de la tecnología empleada para la vigilancia epidemiológica en el campo de las enfermedades cardiovasculares. La información arrojada por el estudio ayudará a la comunidad científica a entender mejor la relación entre la enfermedad coronaria y los factores de riesgo que con ella se asocian. En el transcurso de 1999 se publicarán informes individuales sobre la incidencia de enfermedad coronaria y su relación con factores de riesgo determinados.

Quienes deseen obtener más información sobre este tema deben consultar el siguiente sitio de la WorldWideWeb: http://www.who.ch/ (Organización Mundial de la Salud. Heart disease: good and bad news. Comunicado de prensa WHO/60, 24 de agosto de 1998).

\section{Madre ansiosa, niño enfermo: ¿qué hay de cierto?}

En casi todas las sociedades existe la creencia de que el estado anímico de la embarazada puede afectar al desarollo del niño. Ya se ha documentado en algunos estudios que los hijos de madres ansiosas o agobiadas nacen más temprano y tienen menor peso al nacer que los hijos de madres en estado anímico normal. Según los resultados de un estudio muy extenso de casos y controles, la magnitud del efecto del estrés en el peso al nacer fue similar al detectado en los hijos de madres fumadoras.

Son varios los mecanismos que explican la influencia del estado anímico materno en el desarrollo fetal. Pueden ser mayores las concentraciones de hormonas maternas que cruzan la barrera placentaria. También es posible que se vea disminuido el riego sanguíneo que le llega al feto por las arterias uterinas, efecto ampliamente demostrado en primates inferiores. Con el fin de determinar si el mismo efecto se produce en el ser humano, un grupo de investigadores usó la técnica de Doppler a colores para medir el índice de resistencia en las arterias uterinas y para detectar la presencia de muescas en las ondas producidas por el paso de la sangre por esas arterias. Tales muescas indican que hay bastante resistencia al flujo sanguíneo, fenómeno que se ha asociado en estudios anteriores con resultados obstétricos adversos, especialmente retraso del crecimiento fetal y preeclampsia.

A 100 mujeres con una edad gestacional promedio de 32 semanas se les administró dos cuestionarios: el cuestionario autodirigido de Spielberg para la determinación de estados de ansiedad crónicos y en el momento de la indagación, y un cues- 
tionario sencillo para averiguar sus antecedentes de problemas emocionales, si habían sufrido alguna pena personal en los últimos 3 meses, y si consumían alcohol. El análisis estadístico de estos resultados y los del Doppler reveló una asociación significativa entre el índice máximo de resistencia y los resultados en ambas partes del cuestionario de Spielberg, así como entre estos últimos resultados y el índice de resistencia medio. Cuando las mujeres se dividieron en dos grupos (usando como punto de corte un puntaje $>40$ en ambas partes del cuestionario de Spielberg), las mujeres en el grupo con estados de ansiedad intensos tuvieron una velocidad uterina mucho menor que las mujeres menos ansiosas, bien fuese en términos del índice de resistencia medio, del índice de resistencia máximo, o de la presencia de muescas en las ondas Doppler.

Estos resultados sugieren una posible asociación epidemiológica entre los estados de ansiedad maternos durante el embarazo y los patrones observados de bajo peso al nacer, y entre el medio intrauterino y la presencia de trastornos de salud en el niño después de que nace. (Teixeira JMA, Fisk NM, Glover V. Association between maternal anxiety in pregnancy and increased uterine artery resistance index: cohort based study. Br Med J 1999;318(7177): 153-157).

\section{Los genes, el ambiente y el abuso de sustancias}

Se sabe que el alcoholismo ocurre en familias. También hay pruebas cada vez más convincentes de que la narcodependencia es un trastorno familiar y que factores genéticos explican una buena parte de su tendencia a agruparse en familias. Para explorar más a fondo esta posibilidad, se llevó a cabo un estudio controlado en 231 sujetos con dependencia de los opioides, la cocaína, los derivados de la canabina, el alcohol, o varias de estas sustancias; 61 controles; y 1267 parientes cercanos de los sujetos y controles. Los diagnósticos se establecieron sobre la base de una entrevista semiestructurada; una entrevista estructurada sobre los antecedentes familiares del sujeto, su cónyuge y su pariente cercano; o ambos tipos de entrevista. Los resultados de estas fueron revisados a ciegas y de forma independiente por médicos clínicos con una amplia experiencia en la evaluación y el tratamiento de casos de abuso de sustancias.

Se detectó que el riesgo de dependencia era ocho veces mayor en los parientes de sujetos afectados. Fueron muchas las sustancias implicadas, entre ellas los opioides, la cocaína, los derivados de la canabina y el alcohol. Este riesgo aumentado se observó independientemente de la agrupación familiar observada tanto con el alcoholismo como con los trastornos de la sociabilidad. También hubo indicios de agrupación familiar en relación con tipos particulares de sustancias.

La agrupación familiar del abuso de sustancias podría atribuirse a factores genéticos y ambientales. Es posible, por ejemplo, que los genes predispongan a un individuo a la farmacodependencia debido a diferencias individuales en los efectos que ejercen las propias sustancias, ya sea por razones metabólicas, sensibilidad, tolerancia, efectos secundarios y repercusiones afectivas, emocionales, o cognoscitivas. Asimismo, el ambiente familiar podría aumentar el riesgo de dependencia por exposición a sustancias particulares o como resultado de las disfunciones que a menudo son propias de ese ambiente: problemas maritales, pobreza, abuso sexual, físico o emocional, y demás. Se necesitan más estudios controlados y en gemelos para poder definir mejor los patrones familiares que muestran el abuso de sustancias y los trastornos psiquiátricos que conllevan; determinar si las vías de transmisión genética tradicionales se aplican a los estados de dependencia; determinar por qué el patrón de transmisión varía según el sexo; y dilucidar el papel que desempeñan los genes y el medio ambiente en la narcodependencia. (Merikangas KR, Stolar M, Stevens DE, Goulet J, Preisig MA, Fenton B, et al. Familial transmission of substance use disorders. Arch Gen Psychiatry 1998;55:973-976).

\section{Los traumatismos intracraneanos y el tiempo hasta el inicio de la enfermedad de Alzheimer}

Sigue siendo objeto de discusión si los traumatismos cerebrales son o no un factor de riesgo de enfermedad de Alzheimer. Las pruebas ofrecidas por estudios publicados son contradictorias. Si bien es cierto que estos estudios, en su mayoría, notifican razones de probabilidades (odds ratios) mayores de 1 , muy pocas de estas razones han sido estadísticamente significativas. Un metaanálisis de siete estudios controlados ha arrojado una asociación entre la enfermedad de Alzheimer y los traumatismos cerebrales solamente en el sexo masculino. No obstante, el estudio de una cohorte de 821 personas no reveló ninguna asociación de este tipo. Aunque es posible que estas dudas no se puedan resolver en el futuro próximo, se ha postulado que podría haber una asociación entre sufrir un traumatismo cerebral y el momento en que posteriormente se presenta la enfermedad de Alzheimer. Según esta hipótesis, las personas que por motivos aún desconocidos están en mayor riesgo de padecer dicha enfermedad, un traumatismo cerebral puede acelerar el comienzo de la demencia. Esta hipótesis ha sido examinada en un estudio epidemiológico longitudi- 
nal que abarcó buena parte de la población de Rochester, Minesota, Estados Unidos de América, y del condado vecino de Olmsted.

El lapso esperado normalmente hasta la aparición de la enfermedad de Alzheimer se calculó con los datos obtenidos de otra cohorte de pacientes con esa afección pero sin antecedentes de traumatismo cerebral. Los autores encontraron que, de los 1283 casos de traumatismo cerebral hallados, 31 a la larga padecieron de enfermedad de Alzheimer, número cercano al esperado (razón de incidencias estandarizada $=1,2$; intervalo de confianza de $95 \%$ : $0,8 \%$ a $1,7 \%$ ). No obstante, el tiempo transcurrido entre el traumatismo y el comienzo de los síntomas fue menor que el esperado en personas que no habían sufrido ningún traumatismo (lapso mediano: 10 años frente a $18 ; P=0,015)$. Estos resultados sugieren que un traumatismo intracraneano puede acelerar el comienzo de la enfermedad de Alzheimer en individuos que ya están en riesgo de tener esta afección.

El estudio no está libre de sesgos. Es posible, por ejemplo, que las personas hayan sufrido un traumatismo precisamente debido a una manifestación temprana muy sutil de la enfermedad de Alzheimer. Tampoco se tuvieron en cuenta en el análisis algunos factores genéticos que se consideran predisponentes, como la presencia del genotipo propio de la apolipoproteína E. Los resultados del estudio coinciden, sin embargo, con los de estudios anteriores que han revelado que la interacción entre un traumatismo intracraneano y otros factores de riesgo se traduce en un inicio de los síntomas. Aún no se ha dilucidado el mecanismo causal de este fenómeno, pero se especula, entre otras cosas, la posible mediación del amiloide $\beta$, que es el componente principal de las placas cerebrales características de la enfermedad de Alzheimer. Algunos investigadores han encontrado una asociación entre los traumatismos intracraneanos y la deposición ulterior de esta sustancia en el tejido cerebral lesionado. Ello podría servir de pista para investigar más a fondo las posibles causas del fenómeno aquí descrito. (Nemetz PN, Leibson C, Naessens JM, Beard M, Kokmen E, Annegers JF, et al. Traumatic brain injury and time to onset of Alzheimer's disease: a population-based study. Am J Epidemiol 1999;149(1):32-39.)

\section{Muertes de origen coronario: ¿mienten los certificados de defunción?}

En todo país, los certificados de defunción son una importante fuente de datos para estudios epidemiológicos y clínicos y para sacar las estadísticas nacionales de morbilidad y mortalidad. En los certificados se anotan las causas directas de la defunción, así como las que se consideran subyacentes o contribuyentes, y sobre esa base se determina el panorama a escala nacional. Tal es el caso, por ejemplo, de la enfermedad coronaria, que se considera la causa de muerte más frecuente en los Estados Unidos de América y en muchos países desarrollados, según los datos de los certificados de defunción.

El problema radica en que mucho depende del criterio subjetivo del médico que llena el certificado. Con el fin de examinar la fiabilidad de los certificados en que la enfermedad coronaria figura como causa de defunción subyacente, un grupo de investigadores usó los datos de defunción recogidos durante el famoso estudio de Framingham para medir la sensibilidad, especificidad y los valores predictivos positivo y negativo del certificado de defunción. Se usó como patrón de referencia la causa de defunción subyacente declarada por un panel de tres médicos.

Se encontró un total de 2683 muertes y en 942 de ellas la enfermedad coronaria se había indicado como causa subyacente en el certificado de defunción. En opinión del panel de médicos que revisó los certificados, la enfermedad coronaria era la causa de muerte subyacente en solamente 758 de estos casos. El certificado mostró una sensibilidad de $83,8 \%$, un valor predictivo positivo de $67,4 \%$, una especificidad de $84,1 \%$ y un valor predictivo negativo de $92,9 \%$ en lo que respecta a la enfermedad coronaria.

El certificado de defunción atribuyó a enfermedad coronaria $51,2 \%$ de 242 muertes cuya causa no pudo ser determinada por el panel de médicos. En términos comparativos, el certificado atribuyó a ese trastorno $24,3 \%$ defunciones adicionales en general y por lo menos $50 \%$ defunciones adicionales en personas mayores de 85 años. Aun cuando se excluyeron del análisis las defunciones cuya causa no pudo ser determinada por el panel de médicos, siguió siendo mayor el número de defunciones atribuidas a enfermedad coronaria en los certificados (7,9\% en general y $4,3 \%$ en el grupo de edad más avanzada).

Lo anterior sugiere la posibilidad de que la enfermedad coronaria esté sobrerrepresentada como causa de muerte en los Estados Unidos de América y de que las estadísticas nacionales, que se basan en el contenido de los certificados de defunción, estén sobreestimando la frecuencia general del trastorno en $7,9 \%$ a $24,3 \%$ y hasta en $200 \%$ su frecuencia en personas de edad avanzada. (Lloyd-Jones DM, Martin DO, Larson MG, Levy D. Accuracy of death certificates for coding coronary heart disease as the cause of death. 1998;129(12): 1020-1026). 


\section{Factores que influyen en la edad de la menopausia}

La menopausia, que marca el cese permanente de la menstruación, se acompaña de cambios hormonales cuyo impacto fisiológico es grande y permanente. Las mujeres posmenopáusicas tienen un riesgo aumentado de enfermedad coronaria y accidentes cerebrovasculares, fracturas y osteoporosis. En cambio, la menopausia temprana se ha asociado en numerosos estudios con un menor riesgo de cáncer de mama y endometrio. La edad en que se produce la menopausia, ya sea natural o quirúrgica, es, indudablemente, uno de los factores que más influyen sobre el riesgo que tiene una mujer de padecer estas enfermedades durante su vida. Se han efectuado numerosos estudios al respecto y los resultados no han arrojado conclusiones definidas, con la única excepción del consumo de cigarrillos, que invariablemente ha mostrado una relación inversa con la edad en que se presenta la menopausia.

Los factores asociados con la edad de la menopausia natural son de interés porque pueden ayudar a esclarecer los mecanismos que explican la influencia de la menopausia sobre la aparición de ciertas enfermedades crónicas. En un estudio reciente se exploraron las siguientes variables: tabaquismo, antecedentes reproductivos (menarca y paridad), origen étnico, religión y peso corporal. El estudio se efectuó en 4694 mujeres premenopáusicas (34 a 61 años de edad) que fueron observadas prospectivamente durante un período de 5,4 años. En total, 2035 llegaron a la menopausia durante el período de observación, a una edad promedio de 51,3 años. Las mujeres que eran fumadoras activas tuvieron la menopausia 0,75 años más temprano que las que nunca habían fumado. Las que fumaban más de 10 cigarrillos al día tuvieron un riesgo $40 \%$ veces mayor de tener la menopausia a una edad más temprana. En cambio, las mujeres con tres hijos o más tuvieron la menopausia 0,86 años después que las nulíparas, y las mujeres judías, 0,66 años después que las católicas. También se observó un aumento moderado en la edad de la menopausia en mujeres con un mayor índice de masa corporal.

El presente estudio se destaca por ser el primero en establecer una asociación epidemiológica entre la edad de la menopausia natural y factores ajenos al tabaquismo. No obstante, estos resultados deben interpretarse con cautela, ya que se desconocen los mecanismos que explican las asociaciones observadas. Una de las limitaciones del estudio es que la muestra no fue representativa de la población en general, puesto que se compuso principalmente de mujeres blancas y de buen nivel educativo que acudieron voluntariamente a hacerse la mamografía de rutina. Aun teniendo en cuenta esta y otras limitaciones, el estudio sugiere que la asociación ya conocida entre la edad de la menopausia y el riesgo de enfermedad coronaria, ciertos cánceres y las fracturas de origen osteoporótico puede verse alterada en alguna medida por estos factores. (Ikuku K, Toniolo P, Akhmedkhanov A, Koenig KL, Shore R, Zeleiuch-Jacquotte A. Prospective study of factors influencing the onset of natural menopause. J Clin Epidemiol 1998;51(12):1271-1276).

\section{Una copita diaria: efecto protector contra accidentes cerebrovasculares isquémicos}

Varios estudios han hallado que el consumo moderado de bebidas alcohólicas puede tener un efecto protector contra el riesgo de sufrir un infarto del miocardio, pero aún no se ha podido llegar a la misma conclusión con respecto a los accidentes cerebrovasculares. Aunque numerosos estudios han apuntado hacia una asociación directa entre el consumo de alcohol y el riesgo de un accidente cerebrovascular hemorrágico, los resultados han sido contradictorios en el caso de accidentes cerebrovasculares isquémicos, que constituyen el $80 \%$ del total. En este último caso algunos estudios han detectado más bien un posible efecto protector en ciertas poblaciones, pero no en otras, quizá debido a la influencia de factores raciales. Con el fin de examinar esta relación en personas de diverso origen étnico y racial, de 1993 a 1997 se llevó a cabo un estudio poblacional controlado en una población multiétnica compuesta de personas mayores de 40 años en un barrio de Manhattan, Nueva York, Estados Unidos de América. Los casos fueron pacientes que sufrieron un primer accidente cerebrovascular isquémico durante el período de estudio; los controles fueron elegidos aleatoriamente por contacto telefónico. Ambos grupos fueron sometidos a exámenes físicos y de laboratorio y entrevistados mediante el uso de un instrumento adaptado del Sistema de Vigilancia de Factores de Riesgo Conductuales (Behavorial Risk Factor Surveillance System) de los Centros para el Control y la Prevención de Enfermedades en Atlanta, Georgia, Estados Unidos. El consumo de alcohol se cuantificó mediante entrevistas personales estructuradas y se realizaron estudios internos para determinar la validez del instrumento y la confiabilidad de las respuestas dadas por personas en lugar del enfermo.

La muestra final se compuso de 677 personas con un primer accidente cerebrovascular isquémico. Se encontró que el consumo moderado de alcohol, es decir, un consumo máximo de 2 tragos al día, tiene un efecto protector significativo contra los ac- 
cidentes cerebrovasculares de tipo isquémico aun después de ajustar los datos por presencia de enfermedad coronaria, hipertensión, diabetes, tabaquismo activo, índice de masa corporal y nivel educativo. Este efecto protector se observó en personas de todas las edades y de ambos sexos, así como en personas de raza blanca y negra y de origen hispano. En un modelo cuadrático del riesgo de accidentes cerebrovasculares, se observó un aumento estadísticamente significativo del riesgo de sufrir un accidente isquémico en personas que consumían siete bebidas o más diariamente (razón de posibilidades, 2,96; IC95\%: 1,05 a 8,29).

En resumen, el consumo moderado de alcohol mostró una asociación independiente con un riesgo menor de sufrir un accidente cerebrovascular isquémico, mientras que el abuso del alcohol se asoció con un riesgo aumentado en la población urbana multiétnica de adultos mayores que integró la muestra. Estos datos apoyan las recomendaciones emitidas por la Asociación Nacional de Accidentes Cerebrovasculares (National Stroke Association Stroke Prevention Guidelines) en torno a los beneficios de consumir bebidas alcohólicas con moderación. (Sacco RL, Elkind M, Boden-Albala B, Lin IF, Kargman D, Hauser WA, et al. The protective effect of moderate alcohol consumption on ischemic stroke. JAMA 1999;281(1):53-60).

\section{El yoga mitiga algunos síntomas del síndrome del túnel carpiano}

El síndrome del túnel carpiano, afección de origen ocupacional que se produce por hacer movimientos repetitivos con las manos, afecta a buena parte de la población. Se trata de una afección debilitante con efectos económicos adversos, tanto para el paciente como para la empresa que lo emplea, debido a la pérdida de productividad y días laborales y al elevado costo de la atención médica que requiere. El tratamiento suele consistir en la inmovilización del brazo, el uso de antiinflamatorios, la administración de inyecciones periódicas y hasta la cirugía, pero en muchos casos estas medidas no son muy eficaces para aliviar los síntomas.

El yoga y otras técnicas de relajamiento se han usado para aliviar los síntomas musculoesqueléticos, pero han faltado ensayos clínicos que exploren la utilidad de estas prácticas. Con este fin un grupo de investigadores recientemente realizó un estudio controlado prospectivo a simple ciego en un centro geriátrico y una planta industrial. Participaron en el estudio 42 empleados o personas jubiladas que padecían el síndrome del túnel carpiano. Estas personas fueron divididas en dos grupos: el grupo de los pacientes fue sometido a una intervención a base de yoga; el de los controles fue tratado por inmovilización de la muñeca afectada. La intervención con yoga consistió en 11 posiciones destinadas a fortalecer, estirar y poner en mejor alineamiento las articulaciones de la parte superior del cuerpo, y en ejercicios de relajamiento dos veces por semana durante 8 semanas.

Para determinar la eficacia del yoga frente a la de la inmovilización de la muñeca afectada se investigó si había diferencias en los siguientes parámetros al inicio de la intervención y al cabo de 8 semanas: fuerza en la mano afectada, intensidad del dolor, trastornos del sueño, signos de Phalen y Tinel, y tiempo de conducción motora y sensitiva en el nervio mediano. Se encontró que las personas que practicaron los ejercicios de yoga tuvieron una mejoría estadísticamente significativa en cuanto a la fuerza en la mano afectada y el dolor. Aunque los controles también mostraron mejoría en estos aspectos, la diferencia no fue estadísticamente significativa. Los pacientes mejoraron significatvamente en cuanto al signo de Phalen, pero no mostraron mejoría en lo que respecta al sueño, el signo de Tinel, y el tiempo de conducción sensitiva y motora del nervio mediano. No obstante, en este estudio preliminar el yoga dio mejores resultados que la inmovilización de la muñeca afectada o que la ausencia de un tratamiento. Algunas limitaciones del estudio fueron el pequeño tamaño muestral, la falta de proyectabilidad a la población, el uso de inmovilización física solamente en el grupo control y la ausencia de datos sobre el consumo de medicamentos, el absentismo laboral y el cumplimiento de la inmovilización y otras medidas terapeúticas.

Se cree que las clases de yoga hacen que el paciente sea más consciente de las posiciones corporales y del uso de sus extremidades superiores. Es factible, por lo tanto, que el yoga también ayude a prevenir las recurrencias y el inicio de los síntomas en personas sanas. De hecho, varios sujetos declararon no haber vuelto a tener síntomas durante las 4 semanas posteriores a la intervención. Se necesitan estudios adicionales para determinar si un solo programa de yoga con refuerzos esporádicos basta para proporcionar un alivio duradero. (Garfinkel MS, Singhal A, Katz WA, Allan DA, Reshetar R, Schumacher HR. Yoga-based intervention for carpal tunnel syndrome: a randomized trial. 1998;280 (18):1601-1603). 